\title{
Optimizing phonon space in the phonon-coupling model
}

\author{
V. Tselyaev and N. Lyutorovich \\ St. Petersburg State University, St. Petersburg 199034, Russia
}

J. Speth*

Institut für Kernphysik, Forschungszentrum Jülich, D-52425 Jülich, Germany

P.-G. Reinhard

Institut für Theoretische Physik II, Universität Erlangen-Nürnberg, D-91058 Erlangen, Germany

(Received 27 April 2017; revised manuscript received 28 June 2017; published 18 August 2017)

\begin{abstract}
We present a new scheme to select the most relevant phonons in the phonon-coupling model, named here the time-blocking approximation (TBA). The new criterion, based on the phonon-nucleon coupling strengths rather than on $B(E L)$ values, is more selective and thus produces much smaller phonon spaces in the TBA. This is beneficial in two respects: first, it curbs the computational cost, and second, it reduces the danger of double counting in the expansion basis of the TBA. We use here the TBA in a form where the coupling strength is regularized to keep the given Hartree-Fock ground state stable. The scheme is implemented in a random-phase approximation and TBA code based on the Skyrme energy functional. We first explore carefully the cutoff dependence with the new criterion and can work out a natural (optimal) cutoff parameter. Then we use the freshly developed and tested scheme for a survey of giant resonances and low-lying collective states in six doubly magic nuclei looking also at the dependence of the results when varying the Skyrme parametrization.
\end{abstract}

DOI: 10.1103/PhysRevC.96.024312

\section{INTRODUCTION}

The most often applied approach to collective phenomena in nuclear physics is the random-phase approximation (RPA) [1]. It provides a reliable description of the spectral distribution of multipole excitations from low-energy collective states to the giant resonance region. If one includes the single-particle $(s p)$ continuum we end up with the continuum RPA [2] which allows one to calculate the escape width of giant resonances. In medium and heavy mass nuclei the experimental width however is dominated by the spreading width which involves higher order effects. These may be explicitly two-particle two-hole correlations [3] or the coupling to phonons which also gives rise to a broadening [4-8] of the strength. The most complete formulation of the quasiparticle-phonon coupling has been developed in Ref. [9] which is the basis of the present investigation. This approach is called the quasiparticle time-blocking approximation (TBA). As input one needs $s p$ energies, $s p$ wave functions, and a particle-hole $(p h)$ force. Here we have to distinguished two different approaches. In the first case the $s p$ quantities are taken from a shell model which parameters are adjusted to the experimental $s p$ energies. The $p h$ force is, e.g., modeled as a zero-range, density-dependent interaction, and the corresponding parameters are adjusted to appropriate nuclear structure properties (Refs. [10,11]). In the second case, one starts from an effective Lagrangian or Hamiltonian which allows a fully self-consistent description of nuclear ground state and subsequent dynamics; see Ref. [12] and references therein. Both approaches can equally well

\footnotetext{
*J.Speth@fz-juelich.de
}

be complemented by the TBA to account for complex configuration.

As compared to other treatments of complex configurations (e.g., second RPA [13]), the phonon-coupling method (i.e., TBA) is particularly efficient by confining the complex configurations to a very manageable number of phonons. Thus the key task of the TBA is to select properly the most relevant phonons (i.e., RPA modes). It is obvious that we should chose those phonons which incorporate a large amount of the interaction. These are the collective phonons which consist of a coherent superposition of many $p h$ states. Besides delivering the strongest contributions, using collective phonons is not so much plagued by double counting as predominantly $s p$ excitations do. In the present paper we present a new method of selecting the phonon space for the TBA which is applicable to light and heavy nuclei. This method is presented in Sec. II and compared with previously used selection criteria.

As a preview, Fig. 1 illustrates the effect of the TBA and the impact of phonon space for the example of isovector dipole strength in ${ }^{208} \mathrm{~Pb}$ (details of the method will be explained later). The RPA result resides correctly in the region of the giant dipole resonance (GDR), but has a marked double-peak structure which is at variance with data. The spreading width described with the TBA dissolves the upper peak through substantial broadening and so recovers nicely the experimental one-peak structure. The TBA results are shown for three different choices of phonon space. Formerly, we used the $B(E L)$ strength as a measure of collectivity taking into account only phonons above a certain cutoff value. The curve marked $b_{\text {cut }}=0.2$ stems from this old recipe. In this paper, we will present a new and more selective criterion relying on the phonon-nucleon coupling. We denote this method the $V / E$ criterion and the corresponding cutoff parameter $v_{\min }$. 


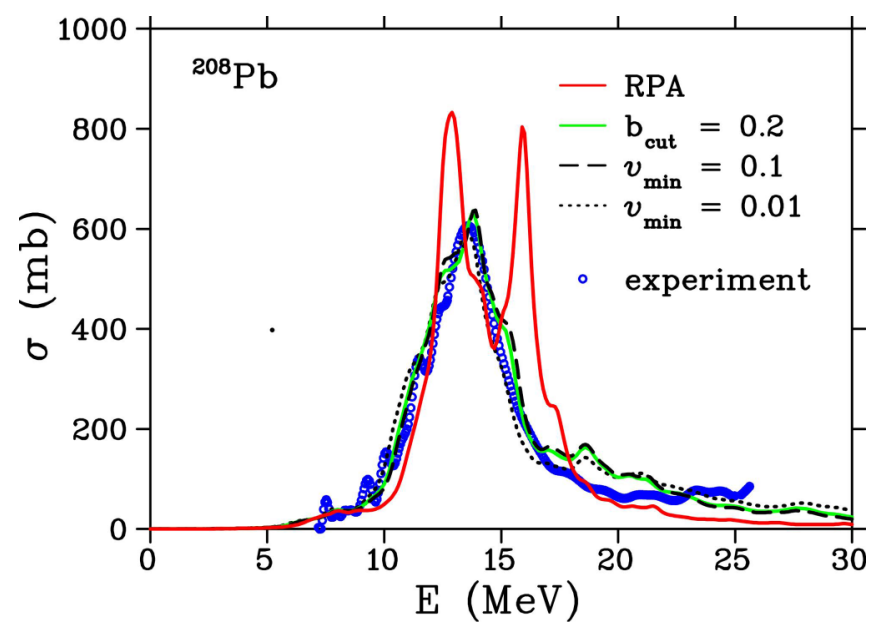

FIG. 1. The giant dipole resonance in ${ }^{208} \mathrm{~Pb}$ calculated using the RPA (red solid line) and the TBA with different selection criteria for phonons: $B$ criterion with $b_{\text {cut }}=0.2$ (green solid line) and $V / E$ criterion with $v_{\min }=0.1$ (black dashed line) and $v_{\min }=0.01$ (black dotted line). The Skyrme parametrization SV-bas was used. Details of the methods and numerical parameters are described in Sec. II. Experimental data from [14].

The curve $v_{\min }=0.1$ yields practically the same results as previously, however, employing many fewer phonons. The dependence on cutoff is indicated with the curve $v_{\min }=0.01$ which makes a slight difference as compared to $v_{\min }=0.1$. In the following, we will discuss in detail the optimal choice of the cutoff parameter.

Section II starts with Sec. II A as a brief summary of the RPA, Sec. II B as one of the TBA, and explains in Sec. II C the criteria for selecting the space of most relevant phonons. The latter subsection is decisive as it provides the formal basis for the new selection criterion from which we show later on that it is more efficient than previous choices. Section III present details of the practical treatment, the continuum-response formalism in Sec. III A, numerical aspects in Sec. III B, and the actual nuclear mean field related to the Skyrme energy-density functional in Sec. IIIC. Section IV addresses the central question, namely, the dependence of the TBA results on the selection of phonons. In Sec. IV A the previously used $B$ criterion which relies on the magnitude of the $B(E L)$ values is compared with the new $V / E$ criterion and in Sec. IV $\mathrm{B}$ we discuss the dependence on the new cutoff parameter $v_{\min }$. Here we demonstrate in several figures that this new selection criterion gives rise to a plateau in nearly all cases. Surprisingly also the energies of the low-lying collective $3^{-}$ resonances, which are the most sensitive quantities in this respect, depend only smoothly on the new parameter. This is connected with the stability conditions [15] introduced in the TBA approach. In Sec. V, we present our results for the giant multipole resonances, giant monopole resonance (GMR), GDR, and giant quadrupole resonance (GQR) for light, medium, and heavy mass nuclei. Three Skyrme parameter sets were used with different effective masses. In Sec. VI, the excitation energies and transition probabilities for the low-lying collective $3^{-}$and $2^{+}$states are compared with the experimental values. In both cases, the influence of the phonons is discussed. Finally, in Sec. VII, we summarize the paper and give an outlook on further improvements.

\section{NEW CRITERION FOR THE SELECTION OF THE PHONONS}

\section{A. Summary of the RPA}

The RPA modes $n$ are characterized by the energies $\omega_{n}$ and transition amplitudes $z_{12}^{n}$ which describe the composition of $n$ from the $p h$ and $h p$ states 12 . These are determined by the eigenvalue equation

$$
\sum_{34} \Omega_{12,34}^{\mathrm{RPA}} z_{34}^{n}=\omega_{n} z_{12}^{n},
$$

where

$$
\begin{aligned}
& \Omega_{12,34}^{\mathrm{RPA}}=\Omega_{12,34}^{(0)}+\sum_{56} M_{12,56}^{\mathrm{RPA}} V_{56,34}, \\
& \Omega_{12,34}^{(0)}=h_{13} \delta_{42}-\delta_{13} h_{42}, \\
& M_{12,34}^{\mathrm{RPA}}=\delta_{13} \rho_{42}-\rho_{13} \delta_{42},
\end{aligned}
$$

$\rho$ is the single-particle density matrix, $h$ is the single-particle Hamiltonian, and $V$ is the amplitude of the residual interaction. In symbolic notation, Eqs. (1) and (2) read

$$
\begin{aligned}
\Omega^{\mathrm{RPA}}\left|z^{n}\right\rangle & =\omega_{n}\left|z^{n}\right\rangle, \\
\Omega^{\mathrm{RPA}} & =\Omega^{(0)}+M^{\mathrm{RPA}} V .
\end{aligned}
$$

The transition amplitudes $\left|z^{n}\right\rangle$ are normalized to

$$
\left\langle z^{n}\left|M^{\mathrm{RPA}}\right| z^{n^{\prime}}\right\rangle=\operatorname{sgn}\left(\omega_{n}\right) \delta_{n, n^{\prime}} .
$$

We will suppose that the RPA is self-consistent (though this is not essential for the subsequent formulas), i.e., that the following relations are fulfilled:

$$
h_{12}=\frac{\delta E[\rho]}{\delta \rho_{21}}, \quad V_{12,34}=\frac{\delta^{2} E[\rho]}{\delta \rho_{21} \delta \rho_{34}},
$$

where $E[\rho]$ is an energy density functional. In the basis diagonalizing the operators $h$ and $\rho$ we have

$$
h_{12}=\varepsilon_{1} \delta_{12}, \quad \rho_{12}=n_{1} \delta_{12},
$$

where $n_{1}=0,1$ is the occupation number. In what follows the indices $p$ and $h$ will be used to label the single-particle states of the particles $\left(n_{p}=0\right)$ and holes $\left(n_{h}=1\right)$ in this basis.

\section{B. Summary of the TBA}

In the TBA, Eq. (1) takes the form

$$
\sum_{34} \Omega_{12,34}^{\mathrm{TBA}}\left(\omega_{v}\right) z_{34}^{v}=\omega_{v} z_{12}^{v},
$$

where

$$
\begin{aligned}
& \Omega_{12,34}^{\mathrm{TBA}}(\omega)=\Omega_{12,34}^{\mathrm{RPA}}+\sum_{56} M_{12,56}^{\mathrm{RPA}} \bar{W}_{56,34}(\omega), \\
& \bar{W}_{12,34}(\omega)=W_{12,34}(\omega)-W_{12,34}(0) .
\end{aligned}
$$


The matrix $W(\omega)$ in Eqs. (11) and (12) represents the induced interaction and is defined in the $p h$ subspace as

$$
W_{12,34}(\omega)=\sum_{c, \sigma} \frac{\sigma F_{12}^{c(\sigma)} F_{34}^{c(\sigma) *}}{\omega-\sigma \Omega_{c}},
$$

where $\sigma= \pm 1, c=\left\{p^{\prime}, h^{\prime}, n\right\}$ is an index of the subspace of $p h \otimes$ phonon configurations, $n$ is the phonon's index,

$$
\begin{aligned}
\Omega_{c} & =\varepsilon_{p^{\prime}}-\varepsilon_{h^{\prime}}+\omega_{n}, \quad \omega_{n}>0, \\
F_{12}^{c(-)} & =F_{21}^{c+) *}, \quad F_{p h}^{c(-)}=F_{h p}^{c(+)}=0, \\
F_{p h}^{c(+)} & =\delta_{p p^{\prime}} g_{h^{\prime} h}^{n}-\delta_{h^{\prime} h} g_{p p^{\prime}}^{n},
\end{aligned}
$$

and $g_{12}^{n}$ is an amplitude of the quasiparticle-phonon interaction. These $g$ amplitudes (along with the phonon's energies $\omega_{n}$ ) are determined by the positive frequency solutions of the RPA equations as

$$
g_{12}^{n}=\sum_{34} V_{12,34} z_{34}^{n}
$$

It is important to note the subtraction of the zero-frequency interaction in the induced interaction $\bar{W}(\omega)$. This serves to confine the induced interaction only to dynamical excitations while the ground state remains unaffected [16]. Moreover, this solves part of the double-counting problem and recovers the stability condition (see [15]).

\section{Selection of the most relevant phonons}

In the self-consistent TBA, in which the relations (8) are fulfilled, we formally have no free parameters in addition to the parameters of the energy density functional. Nevertheless, there is the question of what number and what kind of phonons should be included in the $p h \otimes$ phonon space of the model. This question concerns the problem of convergence with respect to enlarging the $p h \otimes$ phonon subspace. So, it is important to select a small number of phonons producing the strongest coupling between the $p h$ and $p h \otimes$ phonon configurations. Moreover, including only sufficiently collective phonons minimizes violation of the Pauli principle and reduces the problem of double counting connected to the second-order contributions. However, the quest of a well-justified and clear criterion of collectivity is still a matter of debate.

For example, the values of the contributions of the separate $p h$ components (first of all, the main component) of the RPA transition amplitude $z_{12}^{n}$ for a positive frequency state, $\omega_{n}>0$, into the norm (7) can be chosen as this criterion (see, e.g., Refs. $[17,18])$. This is quantified by the quantity $\xi_{n}^{2}$,

$$
\xi_{n}^{2}=\max _{(p h)} \sum_{m_{p}, m_{h}}\left(\left|z_{p h}^{n}\right|^{2}-\left|z_{h p}^{n}\right|^{2}\right),
$$

where $m_{p}$ and $m_{h}$ are the projections of the total angular momentum of the particles and holes, and $(p h)$ denotes the set of the remaining single-particle quantum numbers. For the noncollective phonons the value of $\xi_{n}^{2}$ should be close to 1 . The maximum value of $\xi_{n}^{2}$ for the collective phonons $\left(\xi_{\max }^{2}\right)$ lies typically in the interval 0.5-0.6 [17]. This method estimates the spread of the RPA state over the $1 p 1 h$ configurations, but it does not introduce any energy cutoff of the phonon's basis that would be desirable to limit the phonon subspace. In addition, this criterion does not give information about the magnitude of the particle-phonon coupling for the selected phonons.

Frequently, also in our earlier work, the probability criterion is used in which the phonons are selected according to the values of the reduced probability of the electric transition $B(E L)$ calculated with the transition amplitude $\left|z^{n}\right\rangle$ of the phonon (see, e.g., $[10-12,19])$. This means that only phonons with $b_{n}(E L)=B_{n}(E L) / B(E L)_{\max }>b_{\text {cut }}$ are included in the phonon basis, where $B(E L)_{\max }$ is the maximal $B(E L)$ for the given multipolarity $L$, and $b_{\text {cut }}$ is the cutoff parameter typically ranging from $1 / 10$ to $1 / 5$. In Ref. [19] this criterion is formulated in terms of the phonon's contribution to the energy-weighted sum rule (EWSR). This integral method of the selection of the phonons (we will refer to it as the $B$ criterion) is based on the assumption that the excitation modes having the largest transition probabilities are the most collective ones and should have the strongest coupling to the single-particle states (see discussion at the end of this section). However, the connection of the $B$ criterion to collectivity is, in fact, not so obvious.

Here we suggest another method in which the connection to collectivity and interaction strength becomes more explicit. Let us introduce the average interaction strength in mode $n$ and average $p h$ energy as

$$
\begin{aligned}
\langle V\rangle_{n} & =\left\langle z^{n}|V| z^{n}\right\rangle, \\
\left|\omega_{n}^{(0)}\right| & =\left\langle z^{n}\left|M^{\mathrm{RPA}} \Omega^{(0)}\right| z^{n}\right\rangle .
\end{aligned}
$$

In terms of the basis (9) we have

$$
\left|\omega_{n}^{(0)}\right|=\sum_{p h}\left(\varepsilon_{p}-\varepsilon_{h}\right)\left(\left|z_{p h}^{n}\right|^{2}+\left|z_{h p}^{n}\right|^{2}\right) .
$$

From Eqs. (5) and (7) we obtain

$$
\left\langle z^{n}\left|M^{\mathrm{RPA}} \Omega^{\mathrm{RPA}}\right| z^{n}\right\rangle=\left|\omega_{n}\right| .
$$

From Eqs. (6), (19), (20), (22) and from the property $\left(M^{\mathrm{RPA}}\right)^{2}=1$ in the $p h$ space it follows that

$$
\langle V\rangle_{n}=\left|\omega_{n}\right|-\left|\omega_{n}^{(0)}\right| .
$$

As follows from Eq. (19), the quantity $\langle V\rangle_{n}$ represents the average value of the residual interaction in the RPA state $\left|z^{n}\right\rangle$. The values of $\langle V\rangle_{n}$ can be easily calculated using Eqs. (21) and (23) if the solutions of the RPA equation are known.

The new criterion for selection of phonons (positive frequency: $\omega_{n}>0$ ) is

$$
\left|v_{n}\right|>v_{\min }, \quad v_{n}=\langle V\rangle_{n} / \omega_{n} .
$$

This means that only those phonons will be included in the TBA basis whose dimensionless interaction strength $v_{n}$ exceeds the cutoff value $v_{\min }$. Note that the negative (positive) sign of $v_{n}$ indicates that the residual interaction in the state $\left|z^{n}\right\rangle$ has on average attractive (repulsive) character. We call this selection the $V / E$ criterion in the following.

There are several arguments to justify the $V / E$ criterion. First, from Eqs. (17) and (19) we obtain

$$
\langle V\rangle_{n}=\left\langle z^{n} \mid g^{n}\right\rangle .
$$


In the macroscopic approach (see, e.g., Refs. [20,21]) both the transition amplitudes $z_{12}^{n}$ and the amplitudes $g_{12}^{n}$ are proportional to the dimensionless deformation amplitudes $\beta_{n}$ of the respective vibrational modes. So, in this approach $\left|v_{n}\right| \propto \beta_{n}^{2}$. Therefore, the selection of the phonons with the largest values of $\left|v_{n}\right|$ corresponds to the selection of the low-energy vibrational modes with the largest deformation amplitudes having thus the strongest coupling to the singleparticle states.

Second, we take the point of view that $\left|v_{n}\right|$ is a measure of collectivity. Large values make a large (collective) shift of energy as shown by Eq. (23). Small values correspond to uncorrelated (noncollective) RPA modes which are dominated by what is called a one-loop diagram. But just such uncorrelated RPA modes taken as the phonons produce the second-order contributions in the response function of the TBA which should be eliminated to avoid double counting [22].

Third, the $V / E$ criterion seems to be preferable as compared with the $B$ criterion because it requires no additional assumptions. Note that the $B$ criterion relies on $B(E L)$ values which refer to an external multipole operator and it requires additional cutoff value $L_{\max }^{\text {phon }}$ and multipolarity whereas the $V / E$ criterion is the same for all the multipolarities and automatically eliminates all states with too large values of $L$.

Nevertheless, it is interesting to establish a connection between the $V / E$ and $B$ criteria. Consider a residual interaction $V$ in a separable form as a multipole decomposition (see, e.g., [4]),

$$
V=\sum_{\alpha, L, M} \kappa_{L}^{\alpha}\left|Q_{L M}^{\alpha}\right\rangle\left\langle Q_{L M}^{\alpha}\right|,
$$

where $Q_{L M}^{\alpha}$ are the multipole operators, index $\alpha$ labels different kinds of these operators (electric, magnetic, isoscalar, isovector, etc.), and $\kappa_{L}^{\alpha}$ are the force parameters. If the same $Q_{L M}^{\alpha}$ is taken as the multipole operator for the $B(E L)$ value, the respective reduced probability in the RPA reads

$$
B_{n}\left(\alpha L_{n}\right)=\sum_{M_{n}}\left|\left\langle z^{n} \mid Q_{L_{n} M_{n}}^{\alpha}\right\rangle\right|^{2}
$$

From Eqs. (19), (26), and (27) we obtain

$$
\langle V\rangle_{n}=\sum_{\alpha} \frac{\kappa_{L_{n}}^{\alpha}}{2 L_{n}+1} B_{n}\left(\alpha L_{n}\right) .
$$

Thus the $B(E L)$ values are indeed strongly related to the average interaction strength $\langle V\rangle_{n}$. There are, however, different weight factors which impact the criteria. The $V / E$ criterion employs a weight $\kappa_{L_{n}}^{\alpha}\left(2 L_{n}+1\right)^{-1} \omega_{n}^{-1}$ [if Eq. (28) is fulfilled and the phonon state selects a certain value of $\alpha$ ] while the $B$ criterion is weighted with $B(E L)_{\max }^{-1}$. Different weights create different selectivity and we have yet to see how the two criteria compare in practice. Realistic residual interactions are not strictly separable, but are found often rather close to a sum of separable terms [23]. Thus the above relation maintains some general relevance.

\section{DETAILS OF CALCULATIONS}

\section{A. Basic equations}

Our approach is based on the version of the response function formalism developed within the Green's function method (see [24,25]). Details are described in Refs. [12,26]. The basic calculated quantity is the response $R(\omega)$ which is a solution of the Bethe-Salpeter equation. In the RPA and TBA, it reads

$$
\begin{aligned}
& R^{\mathrm{RPA}}(\omega)=R^{(0)}(\omega)-R^{(0)}(\omega) V R^{\mathrm{RPA}}(\omega), \\
& R^{\mathrm{TBA}}(\omega)=R^{(0)}(\omega)-R^{(0)}(\omega)[V+\bar{W}(\omega)] R^{\mathrm{TBA}}(\omega),
\end{aligned}
$$

where

$$
R^{(0)}(\omega)=-\left(\omega-\Omega^{(0)}\right)^{-1} M^{\mathrm{RPA}}
$$

is the uncorrelated $p h$ propagator. The $p h$ interaction $V$ is defined in Eq. (8) and the induced interaction $\bar{W}(\omega)$ in Eqs. (12) and (13).

Knowledge of the response function allows us to calculate the distribution of the nuclear transition strength $S(E)$ caused by an external field which is represented by a single-particle operator $Q$. It reads

$$
\begin{aligned}
& S(E)=-\frac{1}{\pi} \operatorname{Im} \Pi(E+i \Delta), \\
& \Pi(\omega)=-\langle Q|R(\omega)| Q\rangle,
\end{aligned}
$$

where $E$ is an excitation energy, $\Delta$ is a smearing parameter, and $\Pi(\omega)$ is the (dynamic) polarizability.

\section{B. Details of the numerical treatment}

The two approaches studied here, the RPA and TBA, are realized with the same numerical representation. The $s p$ energies, $s p$ wave functions, and residual $p h$ interaction are obtained from stationary Skyrme-Hartree-Fock (SHF) calculations based on the Skyrme energy functional. All terms of the residual interaction are treated in the RPA and TBA fully self-consistently, according to the formulas given in Ref. [27], but with one exception: the spin-spin terms are omitted. This does not break self-consistency because the terms are not active in the ground state of the double-magic nuclei. The new treatment of the single-particle continuum [12] is used both in the RPA and TBA calculations. There, the RPA and the TBA equations are solved in a discrete basis that simplifies calculations of matrix elements of the residual interaction. However, the uncorrelated $p h$ propagator $R^{(0)}(\omega)$ is constructed with Green's functions properly taking into account the nucleon continuum. Therefore the uncorrelated as well as the correlated propagators do not contain discrete poles at positive energies but a smooth cut.

The box sizes were $15 \mathrm{fm}$ for ${ }^{16} \mathrm{O}$ and ${ }^{40,48} \mathrm{Ca}$ and $18 \mathrm{fm}$ for ${ }^{56} \mathrm{Ni},{ }^{132} \mathrm{Sn}$, and ${ }^{208} \mathrm{~Pb}$. The maximal angular momentum of the $s p$ basis was limited to $l_{\max }^{\mathrm{sp}}=17$ which was found by several tests to be a sufficiently large value. We checked the dependence of the results on the maximum $s p$ energy $\varepsilon_{\max }^{\mathrm{sp}}$. For light $\left({ }^{16} \mathrm{O}\right)$ and medium mass nuclei $(\mathrm{Ca}$ and $\mathrm{Ni})$ we found saturation at $\varepsilon_{\max }^{\mathrm{sp}}=500 \mathrm{MeV}$, and for heavy nuclei ${ }^{132} \mathrm{Sn}$ and ${ }^{208} \mathrm{~Pb}$ at $\varepsilon_{\max }^{\mathrm{sp}}=100 \mathrm{MeV}$. The maximal $s p$ energies 
were thus set as $\varepsilon_{\max }^{\mathrm{sp}}=500 \mathrm{MeV}$ for ${ }^{16} \mathrm{O},{ }^{40,48} \mathrm{Ca}$, and ${ }^{56} \mathrm{Ni}$ and $\varepsilon_{\max }^{\mathrm{sp}}=100 \mathrm{MeV}$ for ${ }^{132} \mathrm{Sn}$ and ${ }^{208} \mathrm{~Pb}$. The phonon basis is restricted by the cutoff $v_{\min }$ or $B(E L)$, and the dependence on the cutoff will be studied in Sec. IV. The maximal phonon energy is $E_{\max }^{\text {phon }}=40 \mathrm{MeV}$. The maximal phonon angular momentum $L_{\max }^{\text {phon }}$ is determined by these conditions. The maximal angular momentum of the quasiparticle-phonon configurations is $L_{\max }^{1 p 1 h \otimes \text { phon }}=27$. These are the standard settings for the following unless explicitly mentioned otherwise. We keep these parameters of representation ( $s p$ space, maximum phonon energy, and maximum angular momentum) fixed at rather large values because we want to concentrate on the trends with the phonon cutoff $v_{\min }$ or $B(E L)$. The question of convergence with these parameters of representation and possible saving at this site will spared for a forthcoming publication.

Each resonance position was characterized by the energy centroid defined as the ratio $E_{0}=m_{1} / m_{0}$ of the first and zeroth energy moments of the corresponding strength $S(E)$. For the GDR (here we considered the photoabsorption cross section) as well as for the GMR and GQR in ${ }^{132} \mathrm{Sn}$ and ${ }^{208} \mathrm{~Pb}$ (here we considered the fraction of the EWSR), the centroids were calculated in the energy windows $E_{0} \pm 2 \delta$ where $\delta$ was the spectral dispersion. To avoid too small energy windows, we used the constraint $\delta>\delta_{\min }$ where $\delta_{\min }=2.5 \mathrm{MeV}$ for the GDR in ${ }^{16} \mathrm{O}, 2 \mathrm{MeV}$ for the GDR in ${ }^{40,48} \mathrm{Ca},{ }^{132} \mathrm{Sn}$, and ${ }^{208} \mathrm{~Pb}$ and for all resonances in ${ }^{56} \mathrm{Ni}$, and $0.5 \mathrm{MeV}$ for the GMR and GQR in ${ }^{132} \mathrm{Sn}$ and ${ }^{208} \mathrm{~Pb}$. The width $\Gamma$ and dispersion for these resonances were defined as

$$
\Gamma=2 \delta \sqrt{2 \ln 2}, \quad \delta=\frac{\int\left(E-E_{0}\right)^{2} S(E) d E}{m_{0}} .
$$

These $E_{0}$ and $\Gamma$ are approximate values of the Lorentzian parameters.

The isoscalar strengths in light nuclei are distributed in large energy ranges and have a complex structure; therefore for these strengths we used the large windows $11<E<40 \mathrm{MeV}$ for GMR and GQR in ${ }^{16} \mathrm{O}, 10<E<30 \mathrm{MeV}$ for GMR in ${ }^{40,48} \mathrm{Ca}$, and $10<E<25 \mathrm{MeV}$ for GQR in ${ }^{40,48} \mathrm{Ca}$. The peak position of the low-lying dipole strength in ${ }^{132} \mathrm{Sn}$ was determined as the energy with the maximum cross section. The resonance widths $\Gamma$ depend slightly on the smearing parameter $\Delta$. In all the RPA and TBA calculations, we used in Eq. (32) $\Delta=400 \mathrm{keV}$. It is questionable to represent the broad and strongly fragmented spectral distribution in ${ }^{16} \mathrm{O}$ by only two numbers (peak energy, width). But it suffices for the purpose of comparison because we handle the experimental data the same way.

\section{Choice of Skyrme parametrization}

From the variety of self-consistent nuclear mean-field models [28], we consider here the Skyrme-Hartree-Fock (SHF) functional; for a detailed description see [28-30]. Its essential features are as follows: The functional depends on a couple of local densities and currents (density $\rho$, gradient of density $\nabla \rho$, kinetic-energy density $\tau$, spin-orbit density $\vec{J}$, current $\vec{j}$, spin density $\vec{\sigma}$, and kinetic spin density $T$ ). All densities and currents exist twofold, for isospin zero and
TABLE I. Nuclear matter properties for the three Skyrme forces used here: incompressibility $K$, isoscalar effective mass $m^{*} / m$, symmetry energy $a_{\text {sym }}$, Thomas-Reiche-Kuhn sum rule enhancement $\kappa_{\text {TRK }}$. Parametrizations are SV-bas, SV-mas07 [32], and SV-m64k6 [35].

\begin{tabular}{lcccc}
\hline \hline & $K(\mathrm{MeV})$ & $m^{*} / m$ & $a_{\text {sym }}(\mathrm{MeV})$ & $\kappa_{\mathrm{TRK}}$ \\
\hline SV-bas & 234 & 0.90 & 30 & 0.4 \\
SV-mas07 & 234 & 0.70 & 30 & 0.4 \\
SV-m64k6 & 241 & 0.64 & 27 & 0.6 \\
\hline \hline
\end{tabular}

isospin 1. It consists of quadratic combinations of these local quantities, corresponding to pairwise contact interactions. In principle, all parameters in front of these contact terms could be density dependent. In practice, density dependence is considered only for the term $\propto \rho^{2}$ (both isospins). Pairing is incorporated by adding a separate pairing functional. The typically 13-14 model parameters are considered as being universal parameters applying throughout the whole nuclear landscape and bulk matter. They are determined by a fit to a large body of experimental data of the nuclear ground state (binding energies, radii, spin-orbit splittings, pairing gaps). For recent examples see [31-33].

Nuclei span a large range in mass number, but a small one in neutron-proton difference. Their ground states are stationary states. This means that the ground-state fits determine predominantly isoscalar static properties and leave some leeway in other respects. Thus there exist many Skyrme parametrizations which perform comparably well in ground-state properties but differ in the less well determined aspects, among them many response properties, equivalent to nuclear matter properties (NMPs). As a consequence, a study using Skyrme forces should employ a couple of different parametrizations to explore the possible variety of predictions. To quantify the variation, we consider the key response properties of the forces in terms of NMPs, i.e., equilibrium properties of symmetric nuclear matter, namely, incompressibility $K$ (isoscalar static), effective mass $m^{*} / m$ (isoscalar dynamic), symmetry energy $a_{\text {sym }}$ (isovector static), and the Thomas-Reiche-Kuhn (TRK) sum rule enhancement $\kappa_{\mathrm{TRK}}$ (isovector dynamic). These four NMPs have a one-to-one relation to nuclear giant resonances in ${ }^{208} \mathrm{~Pb}$ [32]: $K$ to GMR, $m^{*} / m$ to GQR, $\kappa_{\mathrm{TRK}}$ to GDR, and $a_{\text {sym }}$ to dipole polarizability [34]. In order to allow well-defined explorations, the survey [32] provides a series of Skyrme parametrizations with systematically varied NMPs. The present survey aims at exploring the effect of phonon coupling on excitation properties. Here, the response properties are crucial and we take a minimal subset of these systematically varied parametrizations to discriminate robust features from changing ones. Table I lists the chosen parametrizations and their NMPs. SV-bas is the base point of the variation of forces. Its NMPs are chosen such that dipole polarizability and the three most important giant resonances (GMR, GDR, and GQR) in ${ }^{208} \mathrm{~Pb}$ are well reproduced by RPA calculations. SV-mas07 varies the effective mass while keeping the other NMPs fixed. SV-m64k6 was developed in Ref. [35] with the goal to describe, within the TBA, simultaneously the GDR in 


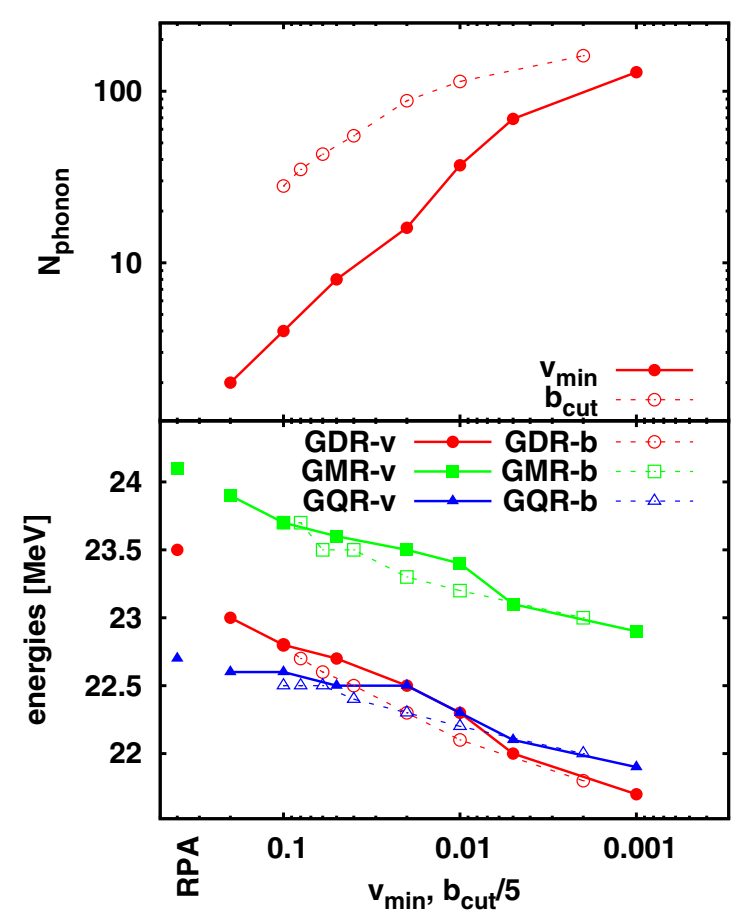

FIG. 2. Trends of TBA results as function of cutoff criterion $v_{\min }$ and $b_{\text {cut }}$ in comparison, computed for ${ }^{16} \mathrm{O}$ with the Skyrme parametrization SV-m64k6. Upper panel: Number of phonon states. Lower panel: Mean energies of the giant resonances where the appendix "-v" indicates computation with $v_{\min }$ cutoff and "-b" $b_{\text {cut }}$ cutoff. On the left side of each panel are the corresponding RPA values displayed, as indicated.

${ }^{16} \mathrm{O}$ and ${ }^{208} \mathrm{~Pb}$. This required pushing up the RPA peak energy in ${ }^{208} \mathrm{~Pb}$ which was achieved by low $a_{\text {sym }}$ in combination with high $\kappa_{\text {TRK }}$. To avoid unphysical spectral bunching for the GDR, a low $m^{*} / m$ was used.

\section{DEPENDENCIES}

In this section, we investigate the dependence of mean energy and width of giant resonances on the cutoff parameter for the phonon space as was introduced in Sec. II. Recall that the crucial ingredient in the phonon-coupling model is the number of active phonons. As already discussed in Sec. II, we propose as selector the parameter $v_{\text {min }}$ which is connected with the collectivity of the phonons. Large $v_{\text {min }}$ exclude automatically phonons which are dominated by one or two $p h$ components only.

\section{A. Comparing $B$ criterion with $V / E$ criterion}

Figure 2 compares mean energies of giant resonances (GRs) (lower panel) and number of active phonons (upper panel) as function of the cutoff parameters $v_{\text {min }}$ and $b_{\text {cut }} / 5$. As one can see, the two criteria give very similar results for the GR energies (lower panel) when scaling $b_{\text {cut }}$ by factor $1 / 5$. However, the number of phonons (upper panel) is very different. The $V / E$ criterion achieves the same GR peak energies with substantially fewer phonons. This indicates that

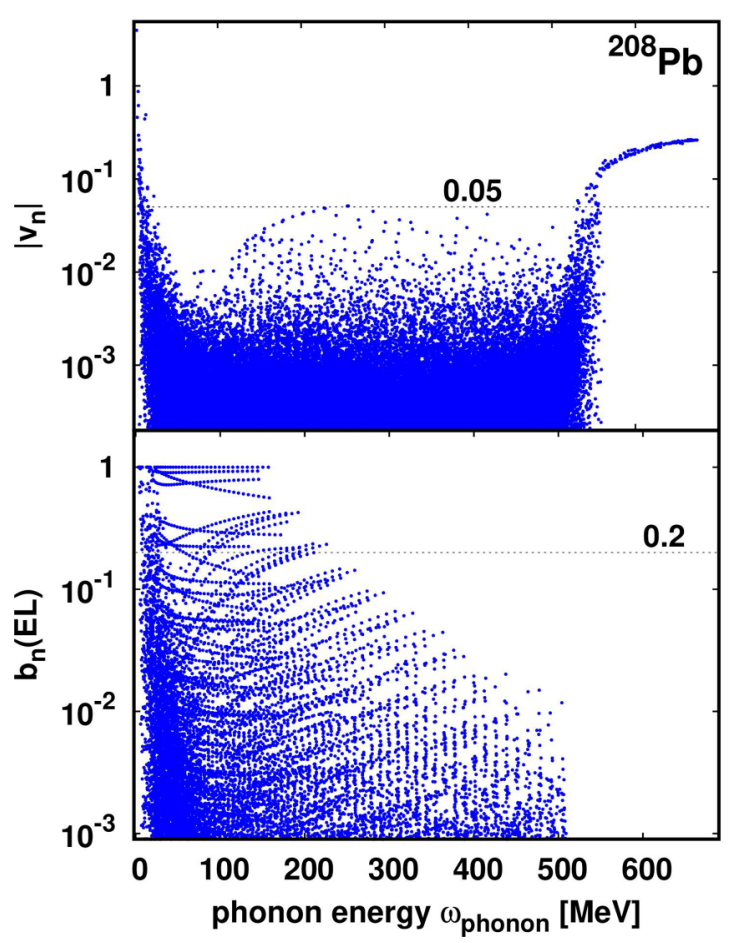

FIG. 3. Scatter plot of the distribution of the two phonon cutoff criteria $\left[v_{n}\right.$ and isoscalar $\left.b_{n}(E L)=B_{n}(E L) / B(E L)_{\max }\right]$ versus phonon energy $\omega_{\text {phon }}$ for ${ }^{208} \mathrm{~Pb}$ computed with the parametrization SV-m64k6 [35]. The dashed horizontal lines indicate the proposed typical cutoffs in each case.

the $V / E$ criterion is more efficient in selecting the relevant phonons.

As another means of comparison, we show in Fig. 3 the distribution of $v_{n}$ values and $b_{n}(E L)$ values as scatter plot versus phonon energy. All values terminate sooner or later near the upper end of $s p$ space which was chosen for the purpose of the test here at $\varepsilon_{\max }^{\mathrm{sp}}=500 \mathrm{MeV}$ (combined with $l_{\max }^{\mathrm{sp}}=45$ ). The strange increase of $v_{n}$ values towards the upper energy is an artifact of the cutoff in $s p$ states and this feature moves with the $\varepsilon_{\max }^{\mathrm{sp}}$. The distributions in the reasonable range below the $\varepsilon_{\max }^{\mathrm{sp}}$ cutoff are dramatically different. The $b_{n}(E L)$ are distributed in a manner which makes it hard to see a preferred cutoff value in $b_{n}(E L)$ and phonon energy. The distribution of $\left|v_{n}\right|$ has the clear advantage that large values of $\left|v_{n}\right|$ appear only at low phonon energies followed by a steep decrease up to $\omega_{\text {phonon }} \approx 50 \mathrm{MeV}$. This automatically confines the relevant phonons to low energy in accordance with intuitive expectation and it picks visibly much less relevant states than the $b(E L)$ criterion. The distribution of $v_{n}$ shows some increase above $\omega_{\text {phon }}=50 \mathrm{MeV}$ reaching an upper limit of $v_{n} \approx 0.05$. This is a particular feature of Skyrme forces and did not happen for the earlier modeling with Migdal interactions. The reason is that the combination of zero range with momentum-dependent coupling induces this increase of coupling with energy. Truly microscopic interactions have finite range and would not show this somewhat unphysical feature. We do not want to reach this region anyway and cut the phonon space at $\omega_{\text {phon }}=40 \mathrm{MeV}$ as usual. However, it is interesting to note that this extra energy 


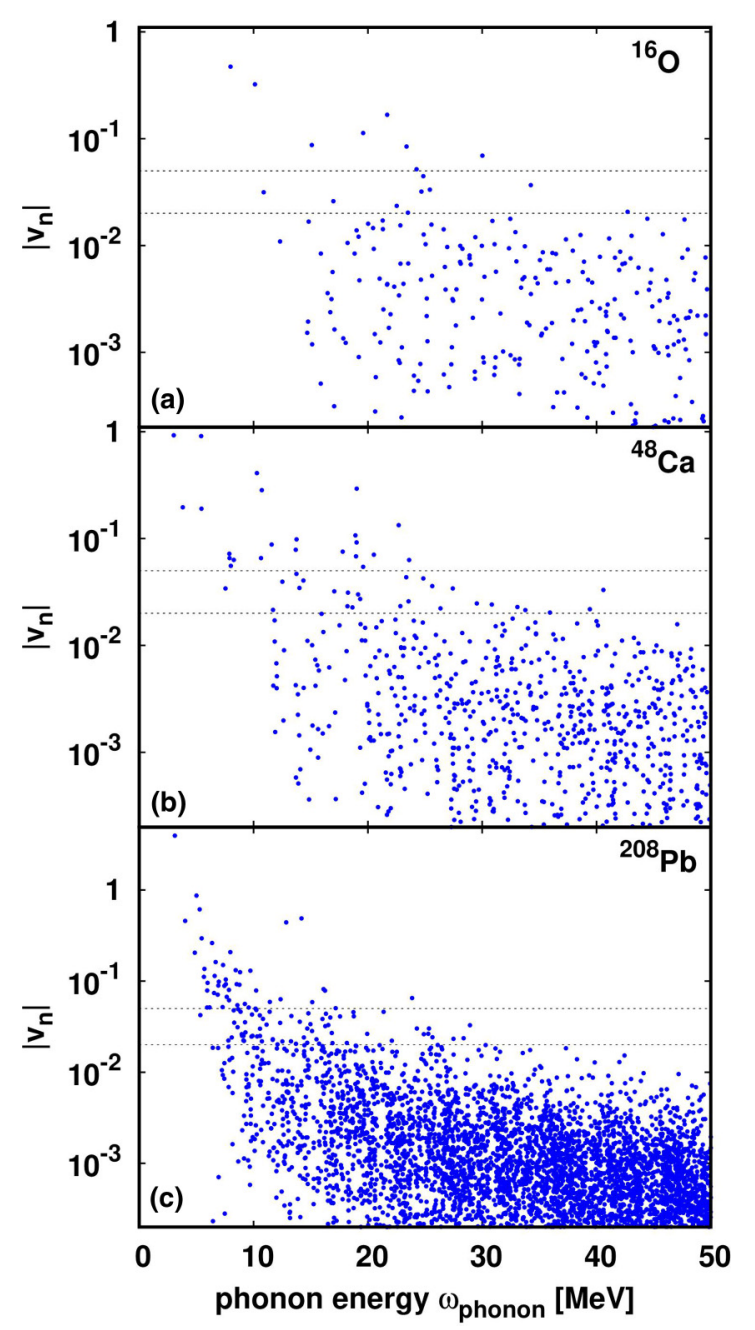

FIG. 4. Scatter plot of the distribution of scaled phonon strength $v_{\text {phon }}=\left|v_{n}\right|$ defined by Eqs. (19) and (24) (blue circles) as function of phonon energy $\omega_{\text {phon }}$ for ${ }^{208} \mathrm{~Pb},{ }^{48} \mathrm{Ca}$, and ${ }^{16} \mathrm{O}$ computed with the parametrization SV-m64k6 [35] and ansp space up to $\varepsilon_{\max }^{\mathrm{sp}}=$ $500 \mathrm{MeV}$. The two dashed horizontal lines indicate the range of reasonable cutoffs $v_{\min }=0.02$ and 0.05 for the selection of phonons in the TBA.

cutoff is redundant if we use as selection criterion $v_{n} \geqslant v_{\min }=$ 0.05. Altogether, Fig. 3 corroborates the findings of Fig. 2 from the detailed perspective of coupling matrix elements.

\section{B. Dependence on the cutoff $v_{\text {min }}$}

The distribution of relative strength of the phonon's states $\left|v_{n}\right|$ defined by Eq. (24) in ${ }^{208} \mathrm{~Pb},{ }^{48} \mathrm{Ca}$, and ${ }^{16} \mathrm{O}$ is shown in Fig. 4. The plot is zoomed to the low phonon energies relevant for the TBA (recall that we set an upper energy limit at $40 \mathrm{MeV}$ ). The two horizontal lines indicate the band of reasonable cutoff values. Below 0.02 we see a quick proliferation of states which we want to avoid. Above 0.05 , the space becomes too dilute. Anything in between should be fine. The upper value $v_{\min }=0.05$ is preferable as the most efficient choice (smallest, set relevant, phonon space). Moreover, it implies automatically the wanted energy cutoff. Furthermore,

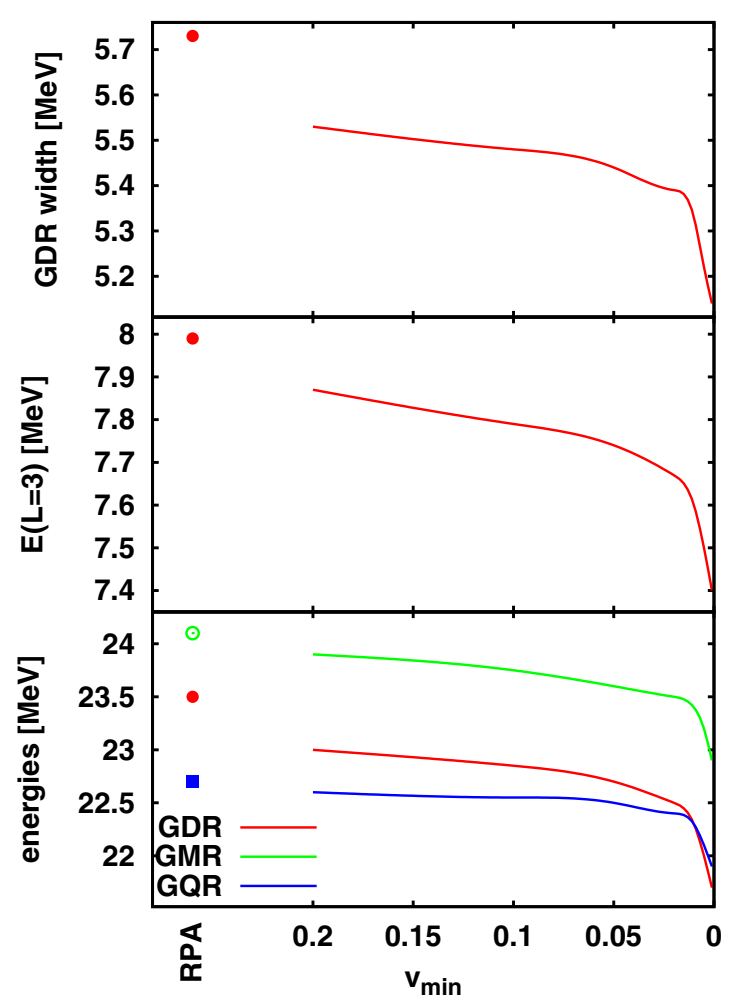

FIG. 5. Mean energies of GR resonances (lower panel), excitation energy of the low-lying $3^{-}$resonance (middle panel), and width of GDR (upper panel) for the case of ${ }^{16} \mathrm{O}$ computed with the force $\mathrm{SV}-\mathrm{m} 64 \mathrm{k} 4$. The results are given as a function of the cutoff criterion $v_{\text {min }}$. On the left side of each panel are the corresponding RPA values displayed, as indicated on the abscissa.

it is gratifying to see that all three nuclei in the figure show the same trends (expect for the different density of states) and thus lead to the same conclusion. To corroborate this choice, we will investigate in the following the dependence of results on resonance energies and width on the choice of cutoff.

Figures 5-7 show the dependence of GR properties and low-lying collective states in ${ }^{16} \mathrm{O},{ }^{48} \mathrm{Ca}$, and ${ }^{208} \mathrm{~Pb}$ on the cutoff parameter $v_{\min }$. The most sensitive dependence on $v_{\text {min }}$ is seen in the light nucleus ${ }^{16} \mathrm{O}$. But even in this case the variation of these key quantities between $v_{\min }=0.2$ and 0.05 (the value which we finally use) is not very large: The energies of GRs are stable and the variation of the width of the GDR as well as the excitation energy of the $3^{-}$state is still moderate. Much less dependence on $v_{\min }$ is seen with increasing mass number $A$. In all cases, we see steep changes starting sooner or later below $v_{\min }=0.05$. Thus we encounter a plateau of robust results within which we can chose pertinent $v_{\text {min }}$. We finally take the lower end of the plateau which complies nicely with the optimum value suggested in the scatter plot in Fig. 4.

\section{GIANT MULTIPOLE RESONANCES}

The RPA and TBA were designed to describe nuclear excitation spectra in the realm of giant multipole resonances [13] 


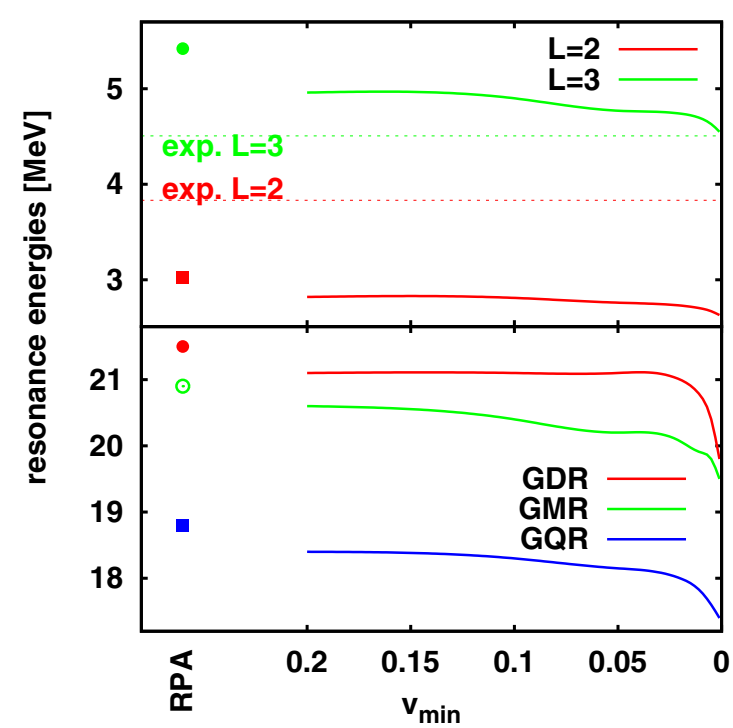

FIG. 6. Mean energies of GR resonances (lower panel) and excitation energy of the low-lying $2^{+}$and $3^{-}$resonances (upper panel) for the case of ${ }^{48} \mathrm{Ca}$ computed with the force SV-m64k4. The results are given as a function of the cutoff criterion $v_{\min }$. On the utmost left side of each panel are the corresponding RPA values displayed, as indicated on the abscissa.

and so GRs are to be the first test case for new developments. We will compare in this section the RPA with TBA results for the three most prominent modes, GDR, GMR, and GQR. In order to demonstrate the influence of the underlying energy functional, we will use three different Skyrme parametrizations as explained in Sec. IIIC. Before going on, let us briefly recall basic properties of GRs. The heavier the nucleus the more concentrated the resonance spectra such that isoscalar GMR and GQR and isovector GDR display one prominent and rather narrow resonance peak. Spectral fragmentation takes over for GMR and GQR towards light nuclei whereas the GDR still remains a compact, though fragmented, resonance. Finally in ${ }^{16} \mathrm{O}$ we observe for GMR and GQR a multipeak structure, which is distributed over a large energy band. As mentioned before to define here a mean energy is somewhat questionable. Nevertheless we can evaluate experimental mean energies the same way which can then be compared with our results.

Figure 8 shows the RPA and TBA results on GRs together with experimental data for the selected nuclei and parametrizations. Although our main emphasis lies on the changes induced by phonon coupling in the step from the RPA to TBA, let us briefly comment on the trends with parametrization. The heavy nucleus ${ }^{208} \mathrm{~Pb}$ being closest to bulk displays a nearly one-to-one correspondence between NMP and GR peak energies [32], namely between GMR and incompressibility, GQR and effective mass, GDR and TRK sum rule enhancement, and dipole polarizability and asymmetry energy. We see this in the upper right panel of Fig. 8: our set of three parametrizations varies $\mathrm{m}^{*} / \mathrm{m}$ and accordingly most changing is the GQR, the set SV-m64k6 varies additionally $\kappa_{\mathrm{TRK}}$, and the GDR peak comes visibly higher. Smaller nuclei gather increasingly surface effects

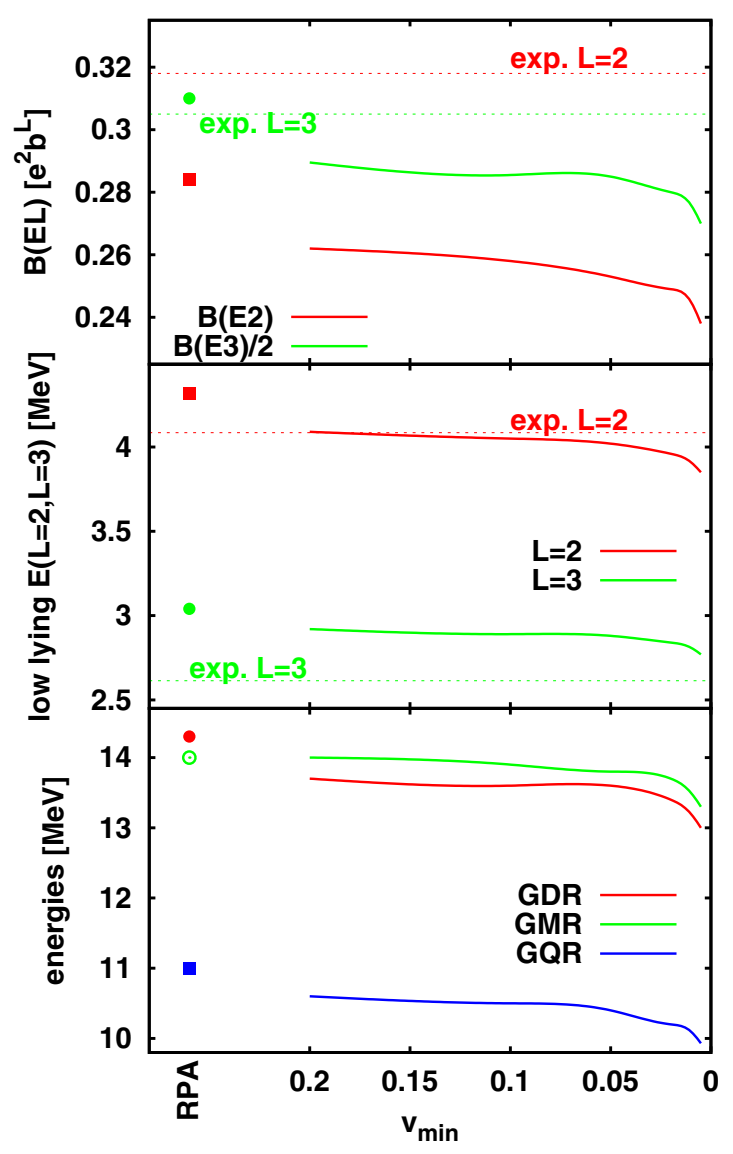

FIG. 7. Mean energies of the giant resonances (lower), excitation energy of the low-lying $3^{-}$state (middle), and their $B(E L) \uparrow$ values (upper) for ${ }^{208} \mathrm{~Pb}$ computed with the force SV-bas. The results are given as a function of the cutoff criterion $v_{\min }$. On the left side of each panel are the corresponding RPA values displayed as indicated on the abscissa.

which, in turn, mixes the dependencies. For example, changing $\mathrm{m}^{*} / \mathrm{m}$ affects all other modes too. Unfortunately, we have to realize that the trend with system size $A$ is not reproduced by only one of the parametrizations. Take the example SV-bas. It is tuned to reproduce GRs in ${ }^{208} \mathrm{~Pb}$ but all three GRs show large deviations for the lightest nuclei in the sample. SV-m64k6 was tuned to cover the GDR in ${ }^{16} \mathrm{O}$ as well as in ${ }^{208} \mathrm{~Pb}$ [35] and it does so, but it fails still for GMR and GQR. The problem remains yet unsolved that the present form of the Skyrme functional cannot yet cover GRs in all nuclei [44]. We will not solve it in this paper. The aim is to check the impact of phonon coupling on the trends which is also an important ingredient in further improving the functional.

Now let us look at effect of phonon coupling in Fig. 8. The step from the RPA to TBA shifts the peak energies to lower energies. The prominent result of this summary view is that this downshift is rather small and constant for all forces and nuclei. It varies only in a small band of $0.5-1.5 \mathrm{MeV}$. The major effect of phonon coupling remains the smoothing of the spectral distributions towards realistic profiles as seen in Figs. 1 and 9. The effect on peak energies is much smaller than the variations with parametrizations. 


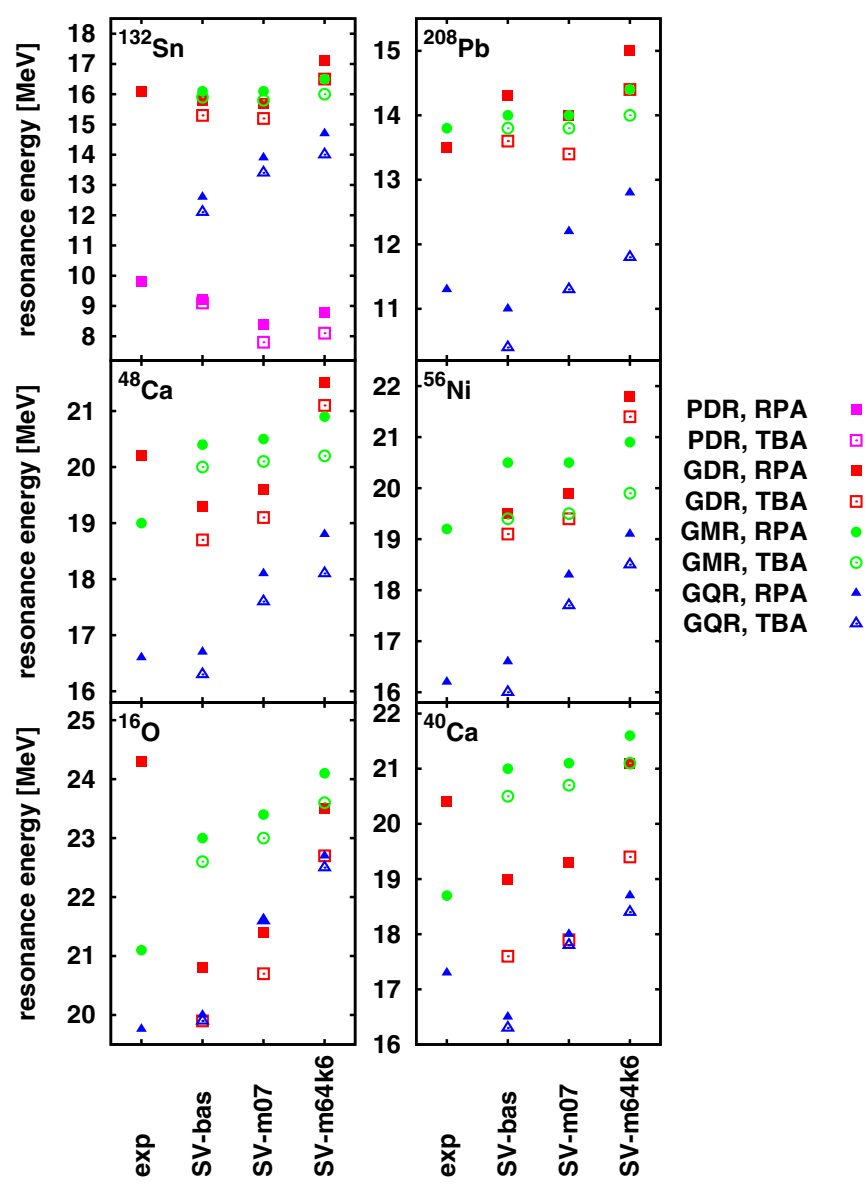

FIG. 8. Mean energies of resonances for three Skyrme parametrizations and six doubly magic nuclei. The experimental data for GMR and GQR in ${ }^{16} \mathrm{O}$ were taken from Ref. [36] and for GDR in ${ }^{16} \mathrm{O}$ from [37], in ${ }^{40} \mathrm{Ca}$ and ${ }^{48} \mathrm{Ca}$ for GDR from [38] and GMR/GQR from [39], in ${ }^{56} \mathrm{Ni}$ for GMR from [40,41] and for GQR from [41], in ${ }^{132} \mathrm{Sn}$ for GDR from [42], in ${ }^{208} \mathrm{~Pb}$ for GDR from [14] and for GMR/GQR from [43].

Thus the solution for the yet unresolved trends with $A$ has to come from improved functionals. However, the fine-tuning of parametrization should stay aware of the small downshift.

The structures of the cross sections of the GR resonances are qualitatively changed by the TBA compared to the RPA. This cannot be seen by comparing the widths of the resonances but one has to look at the detailed cross sections or strength distributions.

Figure 9 shows detailed strength distributions for the three GRs in ${ }^{56} \mathrm{Ni}$ and ${ }^{208} \mathrm{~Pb}$. It demonstrates the impact of phonon coupling in detail. The downshift of peak energy is, though small, quite visible. We see also that the total width is not much affected. It is dominated by spectral fragmentation due to the different energies of the various $p h$ components. This effect, the Landau damping, is already present in the RPA and in general larger than the escape widths due to the coupling to the continuum. the TBA adds what is called spreading width or collisional width [21] which has moderate impact on the total width, but shapes significantly the profile of the spectral distribution by reducing, in most
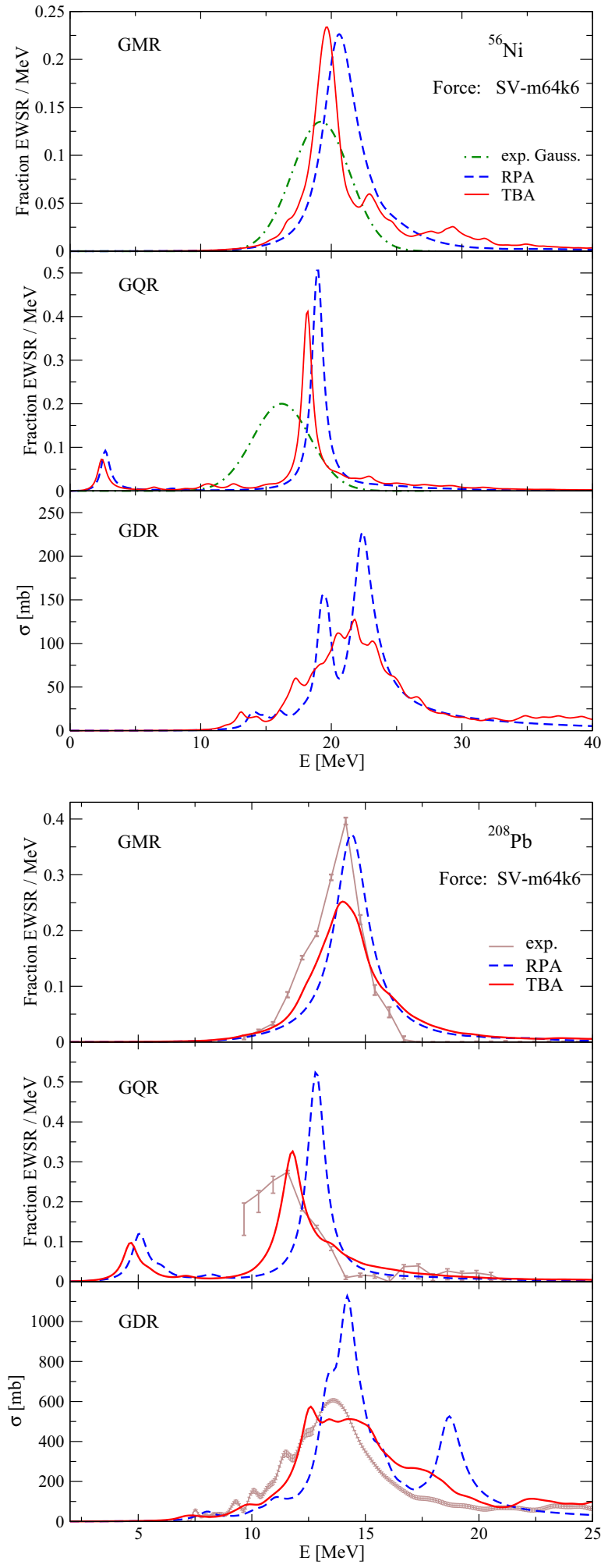

FIG. 9. Strength distributions of giant resonances in ${ }^{56} \mathrm{Ni}$ and ${ }^{208} \mathrm{~Pb}$. Compared are the RPA and TBA results computed using the Skyrme parametrization SV-m64k6. The blue dashed and full red lines represent the RPA and TBA results, respectively. Experimental data (dashed-dotted lines in the upper block) are for ${ }^{56} \mathrm{Ni}$ Gaussians obtained from experimental values of $E_{0}$ and $\Gamma$ [41] and (lines with error bars in the lower block) for ${ }^{208} \mathrm{~Pb}$ from [43] for GMR and GQR and from [14] for GDR. 
TABLE II. Comparison of the energy differences between the spin-orbit $(\Lambda)$ proton partners $1 h_{9 / 2}-1 h_{11 / 2}$ and neutron partners $1 i_{11 / 2}-1 i_{13 / 2}$ and energy gaps $(\Delta)$ between the particle and hole spectra with the experimental data for ${ }^{208} \mathrm{~Pb}$. All the values are given in $\mathrm{MeV}$.

\begin{tabular}{lcccc}
\hline \hline & $\Lambda_{P}$ & $\Lambda_{N}$ & $\Delta_{P}$ & $\Delta_{N}$ \\
\hline${ }^{208} \mathrm{~Pb}$ & & & & \\
SV-bas & 5.22 & 6.83 & 4.09 & 3.86 \\
SV-m64k6 & 5.80 & 7.87 & 4.35 & 5.11 \\
Exp. & 5.57 & 5.84 & 4.21 & 3.43 \\
\hline \hline
\end{tabular}

cases considerably, the height of the main peak. This feature that phonon coupling works predominantly on the detailed profile is new as compared to earlier publications. It is due to the subtraction scheme in Eq. (12) which eliminates double counting of static corrections.

\section{LOW-LYING COLLECTIVE STATES}

\section{A. General considerations}

In all nuclei reported here exists a low-lying collective $3^{-}$ state. As the parity changes from shell to shell one obtains only low-lying $(p h)$ pairs with negative parity. From ${ }^{48} \mathrm{Ca}$ on the spin-orbit splitting is so large that the lower partner of the first unoccupied shell is shifted beneath the Fermi edge in the next lower shell. The coupling of this hole state with the states of the same shell just above the Fermi edge gives rise to low-lying $p h$ pairs with positive parity. For this reasons, in heavy nuclei, the gap between the $p h$ states and the spin-orbit splitting are crucial for the energies of the low-lying states having negative or positive parity. The energy gaps between the particle and hole spectra in ${ }^{208} \mathrm{~Pb}$ shown in Table II are the differences $1 h_{9 / 2}-3 s_{1 / 2}$ for protons and $2 g_{9 / 2}-3 p_{1 / 2}$ for neutrons.

As we can see from Table II in the case of ${ }^{208} \mathrm{~Pb}$ the experimental gaps and the spin-orbit splittings are nicely reproduced by the $\mathrm{SV}$-bas parametrization whereas the results from the SV-m64k6 especially for the neutron data deviate strongly from the experimental values. Therefore we expect that in this case the results for the low-lying $3^{-}$and $2^{+}$states derived with the SV-bas parameters agree much better with the data than the ones obtained with SV-m64k6. In Table III, we show spin-orbit splitting and the energy gaps for ${ }^{40} \mathrm{Ca}$, ${ }^{48} \mathrm{Ca},{ }^{56} \mathrm{Ni}$, and ${ }^{132} \mathrm{Sn}$. Here we compare only the theoretical results for SV-bas with the data. For the spin-orbit splitting the agreement between theory and experimental data is good, with the exception of the proton $1 f$ pair in ${ }^{48} \mathrm{Ca}$. Also the energy gaps between the particle and hole spectra are well reproduced. Here we have to bear in mind that the SV-bas parameter set was not adjusted to any of these quantities but to the usual nuclear matter properties and the monopole, quadrupole, and dipole giant resonances [32].

\section{B. Effective mass, spin-orbit splitting, and phonons}

Figure 10 shows energies and $B(E L) \uparrow$ values of the lowlying collective $3^{-}$and $2^{+}$states in all doubly magic nuclei. The three Skyrme parametrizations have different $m^{*} / m: 0.9$ (SV-bas), 0.7 (SV-m07), and 0.64 (SV-m64k6). As mentioned
TABLE III. Comparison of the spin-orbit splitting $(\Lambda)$ between the proton and neutron partners and the energy gaps $(\Delta)$ between the particle and hole spectra with the experimental data for the doublemagic nuclei ${ }^{40} \mathrm{Ca},{ }^{48} \mathrm{Ca},{ }^{56} \mathrm{Ni}$, and ${ }^{132} \mathrm{Sn}$. All values are given in $\mathrm{MeV}$.

\begin{tabular}{lcccc}
\hline \hline & $\Lambda_{P}{ }^{\mathrm{a}}$ & $\Lambda_{N}{ }^{\mathrm{a}}$ & $\Delta_{P}$ & $\Delta_{N}$ \\
\hline${ }^{40} \mathrm{Ca}$ & & & & \\
SV-bas & 7.06 & 7.45 & 4.99 & 5.10 \\
Exp. & 5.69 & 5.71 & 7.24 & 7.28 \\
${ }^{48} \mathrm{Ca}$ & & & & \\
SV-bas & 7.48 & 7.36 & 5.42 & 2.93 \\
Exp. & 5.08 & 8.75 & 6.18 & 4.80 \\
${ }^{56} \mathrm{Ni}$ & & & & \\
SV-bas & 7.14 & 7.33 & 4.11 & 4.15 \\
Exp. & 7.45 & 7.17 & 6.42 & 6.40 \\
${ }^{132}$ Sn & & & & \\
SV-bas & 5.59 & 7.42 & 5.59 & 4.68 \\
Exp. & 6.13 & 6.75 & 6.13 & 4.94 \\
\hline \hline
\end{tabular}

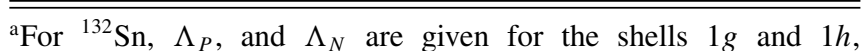
respectively. For all other nuclei, $\Lambda_{P}$ and $\Lambda_{N}$ are given for $1 f$.

above, the low-lying collective $3^{-}$state depends on $m^{*} / m$ in a sensitive and systematic way, the lower $m^{*} / m$ the higher $E\left(3^{-}\right)$because these energies are dominated by the energy gap between the shells with opposite parity which increases with decreasing $m^{*} / m$. The parameter set SV-bas gives for all doubly magic nuclei by far the best results for the energy of the $3^{-}$states. The $E\left(3^{-}\right)$are particularly large for ${ }^{56} \mathrm{Ni}$. This happens because this is the first nucleus in the sample where both shells, protons and neutrons, include the one $s p$ state from the next higher shell which is driven down by spin-orbit splitting. This effectively enhances the energy gap between occupied and unoccupied states of opposite parity.

The energies of the low-lying $2^{+}$states although being enabled by spin-orbit splitting show the same systematic dependence on $m^{*} / m$ because of their strong coupling with the GQR which depends on $m^{*} / m$ as seen above. The theoretical $E\left(2^{+}\right)$in the medium-mass nuclei are slightly too low although the spin-orbit orbit splitting is rather appropriate as seen from Table III. For heavier nuclei, the theoretical value increases relative to data. In all cases, the deviations are, in fact, small. In spite of the differences which we probably pointed out too much, it is astonishing that the Skyrme functionals place the two low-lying states so well although their energies are not fitted and emerge from the subtle interplay of several ingredients.

The effect of phonon coupling (difference between open and filled symbols) is generally small (usually $0.2 \mathrm{MeV}$, occasionally $1 \mathrm{MeV}$ ), smaller than for GRs but relative to the lower excitation energies comparable. The downshift is usually beneficial for coming closer to data in the case of $E\left(3^{-}\right)$. The situation is mixed for $E\left(2^{+}\right)$because the relation of theory to data varies with parametrization and nucleus. The general conclusion remains the same as for GRs: the general trends are not changed by phonon coupling, but the small downshift should be considered when fine-tuning a model. 

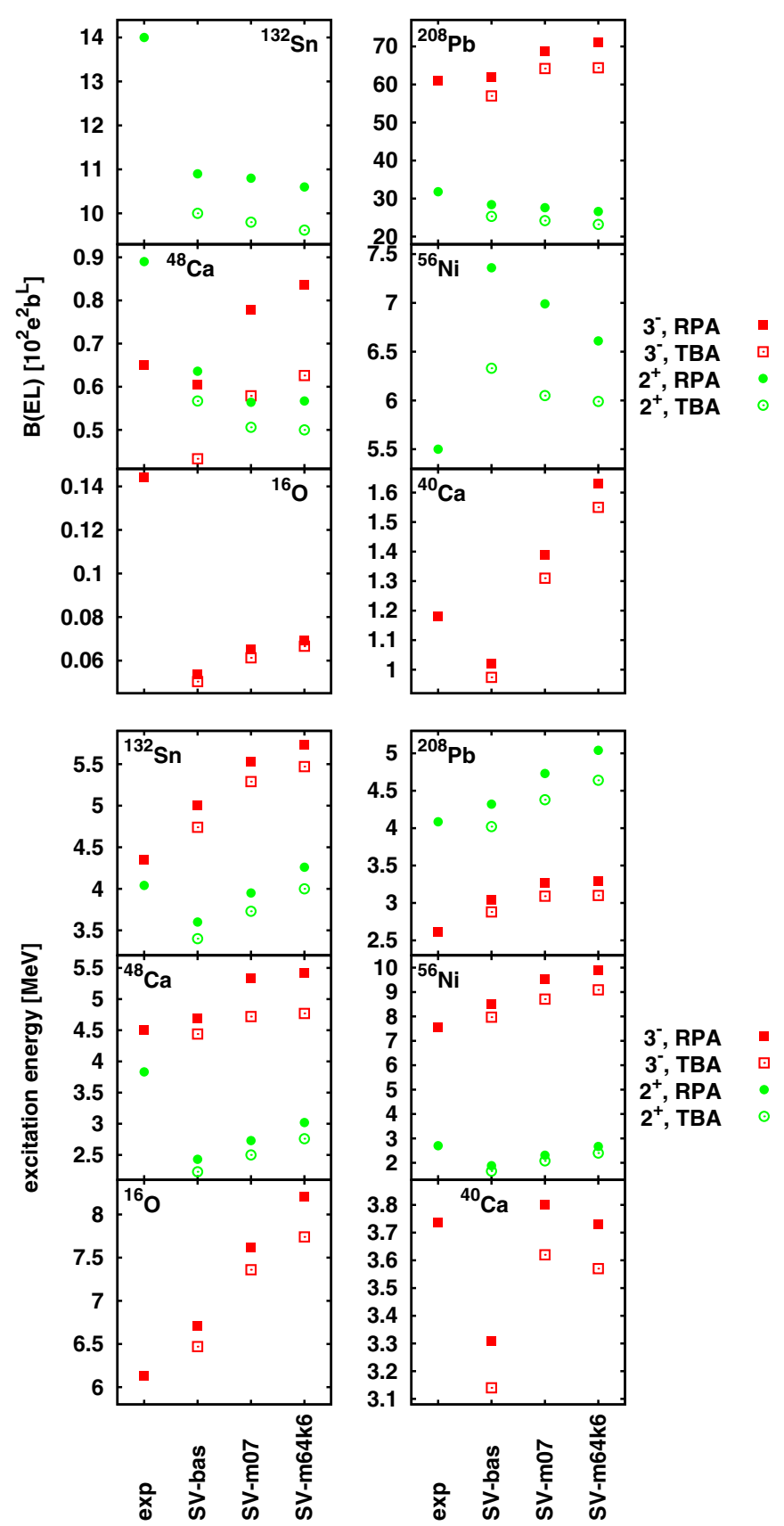

FIG. 10. Energies and $B(E L) \uparrow$ values of low-lying $2^{+}$and $3^{-}$ states for the three selected Skyrme parametrizations and six doubly magic nuclei. The experimental values were taken from the following references: ${ }^{16} \mathrm{O}[45],{ }^{40} \mathrm{Ca}[46],{ }^{48} \mathrm{Ca}[47],{ }^{56} \mathrm{Ni}$ [48], ${ }^{132} \mathrm{Sn} \mathrm{[49],}{ }^{208} \mathrm{~Pb}$ [50].

\section{Transition probabilities $B(E L)$ of the low-lying collective states}

The transition probabilities, $B(E L)$ values shown in the upper panels of Fig. 10, are an even more sensitive test for the quality of a theoretical approach compared with the energies as they test the structure of the wave functions. Figure 10 shows both energies and $B(E L)$ values for the low-lying $2^{+}$ and $3^{-}$collective states together in comparison with data. The agreement is satisfactory. Not only the energies but also $B(E L)$ values in most cases stay within $10 \%$ to $20 \%$ of data. Only the $B(E 3)$ values in ${ }^{16} \mathrm{O}$ deviate by a factor of 3 . This might be connected with the single-particle energies. It has been shown in Ref. [51] that if one starts with the experimental ph energies and includes the excitation energy of the $3^{-}$state in the fitting procedure of the $p h$ force then the $B(E 3)$ value is in good agreement with the data. In the present approach the energy gap between the particle and hole states is $4 \mathrm{MeV}$ smaller compared with the experimental values. As the energy of the $3^{-}$is well reproduced it is obvious that the (self-consistent) $p h$ force is too weak to create collectivity. To a much lesser extent, this can be said of the other cases too. With the exception of the $B(E 2)$ value of ${ }^{56} \mathrm{Ni}$ all $B(E 2)$ values are already in the RPA too small. As mentioned before, the fragmentation of the single-particle strength gives an additional reduction in the TBA.

In this connection one has to bear in mind that in the present approach (like in most other approaches of this kind) correlations beyond RPA are included only in the excited states but not in the ground state. One of the few exceptions is Ref. [52]. Here magnetic dipole states were investigated and in this case, as expected, ground-state correlations beyond RPA give rise to a reduction of the strength. In another investigation [3] the authors treated $2 p 2 h$ correlations consistently in the excited states as well as in the ground state. Here giant resonances were investigated and in the case of the isoscalar the GQR in ${ }^{40} \mathrm{Ca}$ the peak shows, as expected, an enhancement. Unfortunately no results for low-lying collective states were here reported.

Comparing the TBA with RPA we see that phonon coupling always reduces the $B(E L)$ strength, not much but very systematically. This is explained by the fact that, on the one hand, the TBA excitation energies are shifted down with respect to the RPA ones. On the other hand, the RPA-IEWSR (inverse energy-weighted sum rule) is conserved in the TBA with subtraction (see [15]) and therefore the $B(E L)$ values should change in the same direction. The same coincidence of lowering energy together with lowering $B(E L)$ could be observed already in Fig. 7.

\section{SUMMARY}

In this publication we present a new selection criterion for phonons in phonon-coupling models. The criterion relies on the inverse energy-weighted average interaction strength $v_{\text {min }}$ in given RPA modes and so is independent of additional assumptions about external field operators. It is found to sort out (and discard) unambiguously all noncollective states. In this way, it restricts in a natural way the energies and angular momenta of the phonons included in the model space. As a formal motivation, we could show that our new criterion may be compared with the macroscopic method where the low-lying vibrations with the largest deformation parameter give rise to the strongest coupling to the single-particle states. We investigated within the framework of the time-blocking approximation (TBA) the dependence of the results on the magnitude of the new cutoff parameter $v_{\text {min }}$. It is demonstrated that there exists a plateau where the numerical results depend 
only weakly on the parameter $v_{\min }$. From this investigations we extract the quantity $v_{\min }=0.05$.

We applied the newly tuned scheme to a systematic survey of giant resonances as well as of the low-lying collective $2^{+}$ and $3^{-}$resonances in light, medium, and heavy double-magic nuclei where we used three different Skyrme parametrizations to explore the variances of predictions. Thereby we looked at two aspects: first, we studied the effect of phonon coupling in a wide range of nuclei and modes, and second, we looked at the performance in comparison to data. The phonon coupling in the TBA has three effects: a shift of the resonances peak energies, an enhancement of their width, and a smoothing of the spectral distributions. The shift is always downward to lower resonance energies and remains rather small (0.5-1.5 MeV for giant resonance, $0.2-1 \mathrm{MeV}$ for low-lying states). Most importantly, the downshift is for a given mode much the same for all nuclei and forces such that trends (with mass number, with force) are not changed as compared to RPA. The effect on the width is hardly recognizable as this is dominated by RPA's fragmentation width. The largest effect appears in the detailed spectral distributions which are efficiently smoothed such that the TBA profiles come very much closer to the experimental strength distributions.
Where agreement of the results with data is concerned, we have mentioned already the beneficial effect of TBA for spectral distributions. The only small shift of the peak energies leaves the burden of matching resonance positions mainly to the RPA while delivering some fine-tuning from phonon coupling. And here we find again as has been worked out in several earlier RPA studies that the Skyrme energy density functional allows a pertinent description of giant resonances in heavy nuclei but has still unsolved problems with covering the full $A$ dependence of the collective resonances. The present survey gives a direction for further search. The problem of $A$ dependence has first to be resolved roughly within the RPA and then the TBA comes into play when fine-tuning.

\section{ACKNOWLEDGMENTS}

This work has been supported by Contract No. Re322-13/1 from the DFG. N.L. and V.T. acknowledge financial support from the Russian Science Foundation (Project No. 16-1210155). This research was supported by the Computer Center of St. Petersburg University. We thank Dave Youngblood for providing us with experimental data.
[1] D. Bohm and D. Pines, Phys. Rev. 92, 609 (1953).

[2] S. Krewald, J. Kirkholz, A. Faessler, and J. Speth, Phys. Rev. Lett. 33, 1386 (1974).

[3] S. Drożdż, S. Nishizaki, J. Speth, and J. Wambach, Phys. Rep. 197, 1 (1990).

[4] V. G. Soloviev, Theory of Complex Nuclei (Pergamon Press, Oxford, 1976).

[5] J. S. Dehesa, S. Krewald, J. Speth, and A. Faessler, Phys. Rev. C 15, 1858 (1977).

[6] V. I. Tselyaev, Sov. J. Nucl. Phys. 50, 1252 (1989).

[7] S. P. Kamerdzhiev, J. Speth, G. Tertychny, and V. Tselyaev, Nucl. Phys. A 555, 90 (1993).

[8] G. Colo and P. F. Bortignon, Nucl. Phys. A 687, 282 (2001).

[9] V. I. Tselyaev, Phys. Rev. C 75, 024306 (2007).

[10] S. P. Kamerdzhiev, J. Speth, and G. Tertychny, Phys. Rep. 393, 1 (2004)

[11] E. V. Litvinova and V. I. Tselyaev, Phys. Rev. C 75, 054318 (2007).

[12] V. Tselyaev, N. Lyutorovich, J. Speth, S. Krewald, and P.-G. Reinhard, Phys. Rev. C 94, 034306 (2016).

[13] J. Speth and J. Wambach, in Electric and Magnetic Giant Resonances in Nuclei, International Review of Nuclear Physics, Vol. 7, edited by J. Speth (World Scientific, Singapore, 1991), pp. 2-87.

[14] S. N. Belyaev, O. V. Vasiliev, V. V. Voronov, A. A. Nechkin, V. Y. Ponomarev, and V. A. Semenov, Phys. At. Nucl. 58, 1833 (1995).

[15] V. I. Tselyaev, Phys. Rev. C 88, 054301 (2013).

[16] C. Toepffer and P.-G. Reinhard, Ann. Phys. (NY) 181, 1 (1988).

[17] C. A. Bertulani and V. Y. Ponomarev, Phys. Rep. 321, 139 (1999).

[18] E. Litvinova, P. Ring, and D. Vretenar, Phys. Lett. B 647, 111 (2007).
[19] G. Colò, N. Van Giai, P. F. Bortignon, and R. A. Broglia, Phys. Rev. C 50, 1496 (1994).

[20] A. Bohr and B. Mottelson, Nuclear Structure, Vol. 2 (Benjamin, New York, 1975).

[21] G. F. Bertsch, P. F. Bortignon, and R. A. Broglia, Rev. Mod. Phys. 55, 287 (1983).

[22] D. J. Rowe, Nuclear Collective Motion (Methuen, London, 1970).

[23] V. O. Nesterenko, J. Kvasil, and P.-G. Reinhard, Phys. Rev. C 66, 044307 (2002).

[24] S. Shlomo and G. Bertsch, Nucl. Phys. A 243, 507 (1975).

[25] J. Speth, E. Werner, and W. Wild, Phys. Rep. 33, 127 (1977).

[26] N. Lyutorovich, V. Tselyaev, J. Speth, S. Krewald, F. Grümmer, and P.-G. Reinhard, Phys. Lett. B 749, 292 (2015).

[27] N. Lyutorovich, V. Tselyaev, J. Speth, S. Krewald, and P.-G. Reinhard, Phys. At. Nucl. 79, 868 (2017).

[28] M. Bender, P.-H. Heenen, and P.-G. Reinhard, Rev. Mod. Phys. 75, 121 (2003).

[29] J. R. Stone and P. G. Reinhard, Prog. Part. Nucl. Phys. 58, 587 (2007).

[30] J. Erler, P. Klüpfel, and P. G. Reinhard, J. Phys. G 38, 033101 (2011).

[31] S. Goriely, M. Samyn, P. H. Heenen, J. M. Pearson, and F. Tondeur, Phys. Rev. C 66, 024326 (2002).

[32] P. Klüpfel, P. G. Reinhard, T. J. Bürvenich, and J. A. Maruhn, Phys. Rev. C 79, 034310 (2009).

[33] M. Kortelainen, T. Lesinski, J. Moré, W. Nazarewicz, J. Sarich, N. Schunk, M. V. Stoitsov, and S. Wild, Phys. Rev. C 82, 024313 (2010)

[34] W. Nazarewicz, P. G. Reinhard, W. Satula, and D. Vretenar, Eur. Phys. J. A 50, 20 (2014).

[35] N. Lyutorovich, V. I. Tselyaev, J. Speth, S. Krewald, F. Grümmer, and P. G. Reinhard, Phys. Rev. Lett. 109, 092502 (2012). 
[36] Y.-W. Lui, H. L. Clark, and D. H. Youngblood, Phys. Rev. C 64, 064308 (2001).

[37] B. S. Ishkhanov, I. M. Kapitonov, E. I. Lileeva, E. V. Shirokov, V. A. Erokhova, M. A. Yolkin, and A. V. Izotova, preprint INP MSU 2002-27/711, http://www-nds.iaea.org/exfor/exfor.htm

[38] M. R. Anders, S. Shlomo, T. Sil, D. H. Youngblood, Y.-W. Lui, and Krishichayan, Phys. Rev. C 87, 024303 (2013).

[39] V. A. Erokhova, M. A. Elkin, A. V. Izotova, B. S. Ishkhanov, L. M. Kapitonov, E. I. Lileeva, and E. V. Shirokov, Izv. Ross. Akad. Nauk Ser. Fiz 67, 1479 (2003).

[40] S. Bagchi, J. Gibelin, M. Harakeh, N. Kalantar-Nayestanaki, N. Achouri, H. Akimune, B. Bastin, K. Boretzky, H. Bouzomita, M. Caamano, L. Caceres, S. Damoy, F. Delaunay, B. FernandezDominguez, M. Fujiwara, U. Garg, G. Grinyer, O. Kamalou, E. Khan, A. Krasznahorkay, G. Lhoutellier, J. Libin, S. Lukyanov, K. Mazurek, M. Najafi, J. Pancin, Y. Penionzhkevich, L. Perrot, R. Raabe, C. Rigollet, T. Roger, S. Sambi, H. Savajols, M. Senoville, C. Stodel, L. Suen, J. Thomas, M. Vandebrouck, and J. V. de Walle, Phys. Lett. B 751, 371 (2015).

[41] C. Monrozeau, E. Khan, Y. Blumenfeld, C. E. Demonchy, W. Mittig, P. Roussel-Chomaz, D. Beaumel, M. Caamaño, D. Cortina-Gil, J. P. Ebran, N. Frascaria, U. Garg, M. Gelin, A. Gillibert, D. Gupta, N. Keeley, F. Maréchal, A. Obertelli, and J.-A. Scarpaci, Phys. Rev. Lett. 100, 042501 (2008).
[42] P. Adrich, A. Klimkiewicz, M. Fallot, K. Boretzky, T. Aumann, D. Cortina-Gil, U. DattaPramanik, T. W. Elze, H. Emling, H. Geissel, M. Hellström, K. L. Jones, J. V. Kratz, R. Kulessa, Y. Leifels, C. Nociforo, R. Palit, H. Simon, G. Surówka, K. Sümmerer, and W. Walus (LAND-FRS Collaboration), Phys. Rev. Lett. 95, 132501 (2005).

[43] D. H. Youngblood, Y.-W. Lui, H. L. Clark, B. John, Y. Tokimoto, and X. Chen, Phys. Rev. C 69, 034315 (2004).

[44] J. Erler, P. Klüpfel, and P.-G. Reinhard, J. Phys. G 37, 064001 (2010).

[45] D. Tilley, H. Weller, and C. Cheves, Nucl. Phys. A 564, 1 (1993).

[46] J. Chen, Nucl. Data Sheets 140, 1 (2017).

[47] T. Burrows, Nucl. Data Sheets 107, 1747 (2006).

[48] H. Junde, H. Su, and Y. Dong, Nucl. Data Sheets 112, 1513 (2011).

[49] Y. Khazov, A. Rodionov, S. Sakharov, and B. Singh, Nucl. Data Sheets 104, 497 (2005).

[50] M. Martin, Nucl. Data Sheets 108, 1583 (2007).

[51] S. P. Kamerdzhiev, R. J. Liotta, and V. I. Tselyaev, Phys. Rev. C 63, 034304 (2001).

[52] S. P. Kamerdzhiev, J. Speth, G. Tertychny, and J. Wambach, Z. Phys. A 346, 253 (1993). 